Volume 49, Number 4 April 2014
A Monthly Publication of the ACM Special Interest Group on Programming Languages

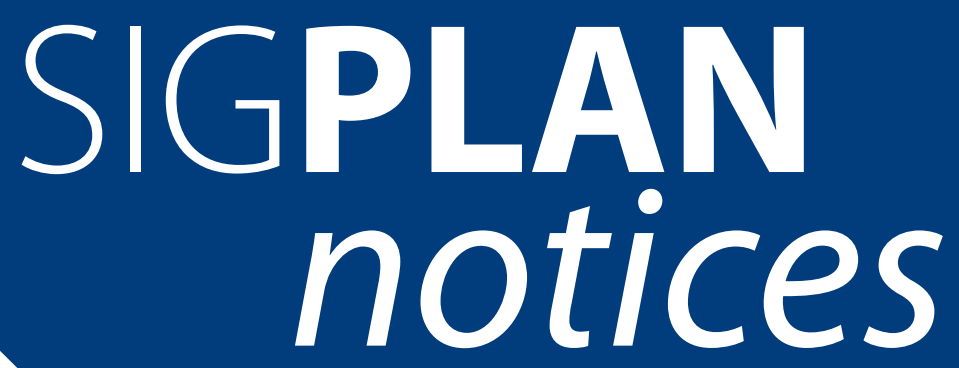

Proceedings of the 19th International Conference on Architectural Support for Programming Languages and Operating Systems (ASPLOS XIX) 
ACM SIGPLAN Notices

A monthly publication of ACM

SIGPLAN

Publications Office

ACM, 2 Penn Plaza, Suite 701, New York, NY 10121-0701, USA

$+1-212-869-7440$

$F A X+1-212-302-9618$

Editor: Andy Gill

Advertising Information:

Jennifer Ruzicka +1-212-869-7440

ACM SIGPLAN Notices is an informal monthly publication of the Special Interest Group on Programming Languages (SIGPLAN) of ACM.

Membership in SIGPLAN is open to ACM Members or associate members for $\$ 25$ (online) and $\$ 130$ (print). Membership for ACM Student members is $\$ 15$ (online) and $\$ 130$ (print). SIGPLAN Notices is available online through the ACM Digital Library. Print members receive a hardcopy of each issue. All members receive the annual SIGPLAN CD, discounts at SIGPLAN events, and may vote in the Group's elections. ACM members of SIGPLAN may serve as officers of the Group.

Institutional or Library subscriptions to ACM SIGPLAN Notices are available for $\$ 110$ per year, and the regular back issues of the Notices may be purchased for \$19 per copy from ACM Headquarters.

Requests for reprints, copies of reports, or references should be sent to authors.

\section{Change of address:} acmcoa@acm.org

Members Services Information: acmhelp@acm.org or +1-212-626-0500

ACM SIGPLAN Notices (ISSN 03621340) is published monthly by ACM, 2 Penn Plaza, Suite 701, New York, NY 10121. The basic annual subscription price is $\$ 130.00$ formembers. Periodicals postage paid at New York, NY 10001 and at additional mailing offices.

POSTMASTER: Send change of Address to ACM SIGPLAN Notices, ACM, 2 Penn Plaza, Suite 701 New York, NY 10121-0701.
SIGPLAN Executive Committee

Chair

Jan Vitek

Dept of Computer Sciences

Purdue University

305 N University Street

West Lafayette, IN 47907, USA

jv@cs.purdue.edu

Vice-Chair for Conferences

Jeremy Gibbons

University of Oxford

jeremy.gibbons@cs.ox.ac.uk

\section{Secretary}

Andrew P. Black

Portland State University

black@cs.pdx.edu

\section{Treasurer}

Cristina Lopes

University of California, Irvine

lopes@ics.uci.edu

\section{Past Chair}

Philip Wadler

University of Edinburgh

wadler@inf.ed.ac.uk

Members at Large:

Derek Dreyer

Max Planck Institute for

Software Systems

dreyer@mpi-sws.org

Sue Eisenbach

Imperial College, London

s.eisenbach@imperial.ac.uk

Mike Hind

IBM

hindm@us.ibm.com

Julia Lawall

INRIA

julia.lawall@lip6.fr

Norman Ramsey

Tufts University

nr@cs.tufts.edu

SIGPLAN Information Director

Andy Gill

andygill@ku.edu

SIGPLAN Program Director

Fran Spinola

spinola@hq.acm.org
Editor ACM SIGPLAN Notices

Andy Gill

2001 Eaton Hall

1520 West 15th Street

Lawrence, KS 66045-7621, USA

andygill@ku.edu

For Notices correspondence: andygill@ku.edu

ASPLOS Steering Committee

Tim Harris

timothy.I.harris@oracle.com

DLS Steering Committee

Robert Hirschfeld

hirschfeld@acm.org

GPCE Steering Committee

Eelco Visser

visser@acm.org

Haskell Steering Committee

Koen Claessen

koen@chalmers.se

ICFP Steering Committee

Peter Thiemann

thiemann@informatik.uni-freiburg.de

ISMM Steering Committee

Vechev Martin

martin.vechev@inf.ethz.ch

LCTES Steering Committee

Bruce Childers

childers@cs.pitt.edu

PLDI Steering Committee

Kathleen Fisher

kfisher@eecs.tufts.edu

POPL Steering Committee

Roberto Giacobazzi

roberto.giacobazzi@univr.it

PPoPP Steering Committee

Calin Cascaval

cascaval@acm.org

SPLASH Steering Committee

Cristina Lopes

lopes@ics.uci.edu

VEE Steering Committee

Dilma da Silva

dilmasilvaatus@us.ibm.com
SIGPLAN's Mission Statement: SIGPLAN, the premier programming languages organization, promotes awareness and advancement of research and practice in programming languages. SIGPLAN accomplishes its mission by sponsoring conferences and publishing newsletters. The SIG also serves as a coordinating body for the volunteers who wish to undertake projects to further the goals of the SIG. 


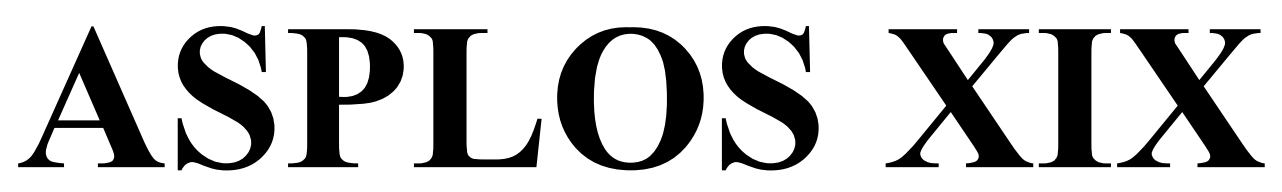

\section{Nineteenth International Conference}

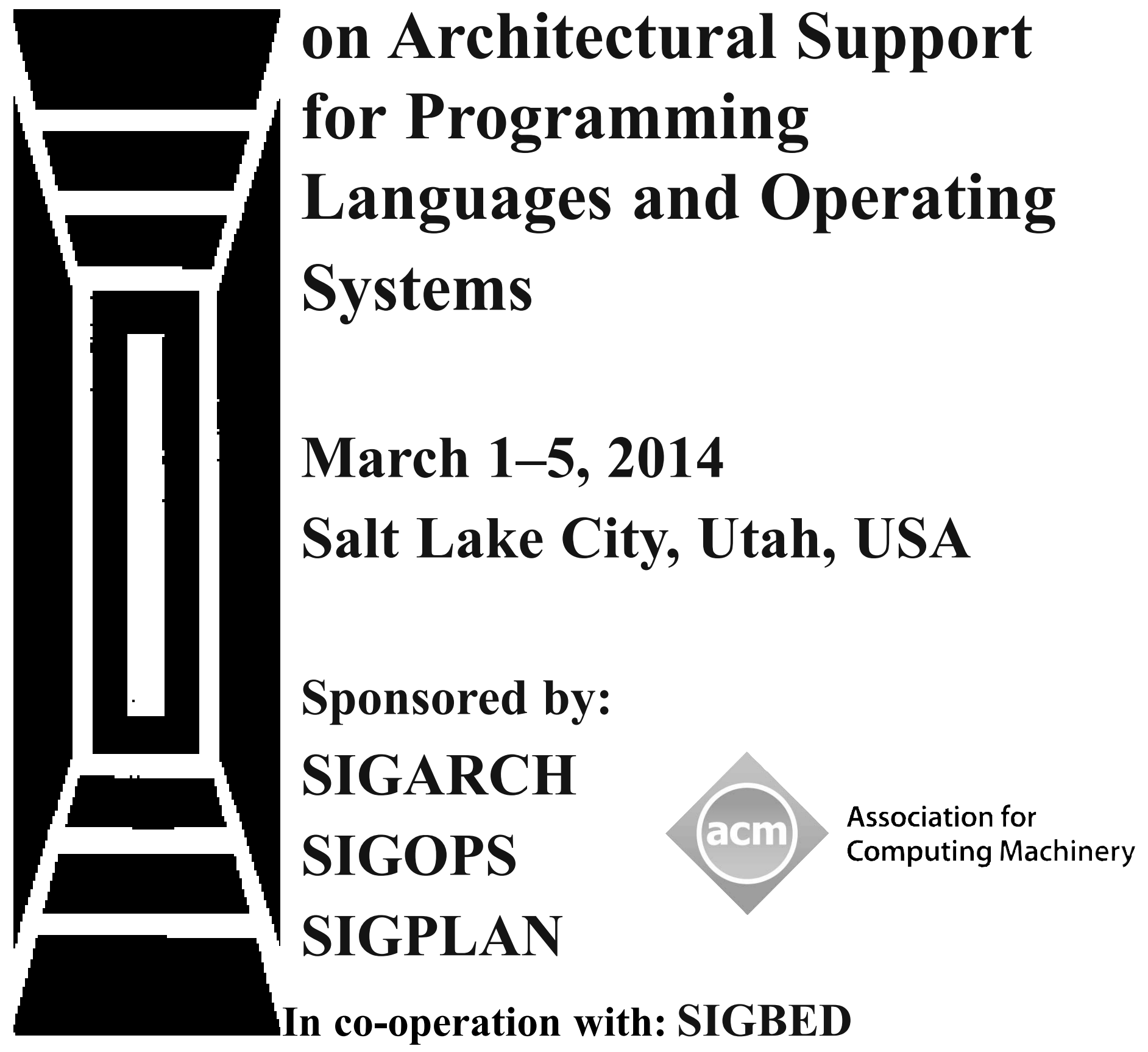

With Support from:

NSF, Microsoft Research, ARM, AMD, Google, Hewlett-Packard, Intel, VMware, Facebook, Oracle Labs, $\mathrm{EMC}^{2}$, and IBM Research 
Association for

Computing Machinery

Advancing Computing as a Science \& Profession

\section{The Association for Computing Machinery 2 Penn Plaza, Suite 701 New York, New York 10121-0701}

Copyright (C) 2014 by the Association for Computing Machinery, Inc. (ACM). Permission to make digital or hard copies of portions of this work for personal or classroom use is granted without fee provided that copies are not made or distributed for profit or commercial advantage and that copies bear this notice and the full citation on the first page. Copyright for components of this work owned by others than ACM must be honored. Abstracting with credit is permitted. To copy otherwise, to republish, to post on servers or to redistribute to lists, requires prior specific permission and/or a fee. Request permission to republish from: permissions@acm.org or Fax +1 (212) 869-0481.

For other copying of articles that carry a code at the bottom of the first or last page, copying is permitted provided that the per-copy fee indicated in the code is paid through www.copyright.com.

Notice to Past Authors of ACM-Published Articles

ACM intends to create a complete electronic archive of all articles and/or other material previously published by ACM. If you have written a work that has been previously published by ACM in any journal or conference proceedings prior to 1978, or any SIG Newsletter at any time, and you do NOT want this work to appear in the ACM Digital Library, please inform permissions@acm.org, stating the title of the work, the author(s), and where and when published.

ISBN: 978-1-4503-2305-5

Additional copies may be ordered prepaid from:

\section{ACM Order Department}

PO Box 30777

New York, NY 10087-0777, USA

Phone: 1-800-342-6626 (USA and Canada)

$+1-212-626-0500$ (Global)

Fax: +1-212-944-1318

E-mail: acmhelp@acm.org

Hours of Operation: 8:30 am - 4:30 pm ET

Printed in the USA 


\section{ASPLOS 2014 General Chairs' Welcome}

It is our great pleasure to welcome you to the 2014 ACM International Conference on Architectural Support for Programming Languages and Operating Systems - ASPLOS-XIX in Salt Lake City, UT on March 1-5, 2014.

This year's ASPLOS finds a home in beautiful Salt Lake City, a thriving technology hub that is surrounded by snow-capped mountains and a handful of geologically diverse national parks. In fact, the large number of technology start-ups in the Salt Lake valley has inspired the moniker "Silicon Slopes". Visitors can choose among a wide array of activities while in town - skiing or snowboarding in one of eight nearby world-class ski resorts, hiking on numerous local trails, visiting Historic Temple Square in downtown, or taking the five-hour drive to national parks in Utah and surrounding states. As the site of the 2002 Winter Olympic games, there are numerous Olympic class facilities nearby. The conference banquet will be held at the Olympic Oval, site of both the long and short track speed skating events. Apollo Anton Ohno appeared on the world stage as the gold medalist in the 1500 meter short-track event in the 2002 Olympics. We hope the banquet will not only provide the usual social opportunity to mingle with colleagues but also give you all the chance to feel some of the spirit that lies behind the Winter Olympic Games.

This year's conference continues the ASPLOS tradition of being the premiere venue for reporting integrative research comprising computer architecture, programming languages, and operating systems. The conference represents the hard work of the organizing committee, reviewers, and in particular the contributing authors. Particular thanks goes to Sarita Adve for the amazing effort she put in to create the technical program. Her organization and diligence in every aspect was simply spectacular. The Program Chair message details her many innovative approaches to improving our peer review process. We also thank the other organizing committee members who worked hard behind the scenes to make this conference possible: Gabe Loh (AMD Research) for his efforts on fund-raising; Venkatesh Akella (UC Davis) for organizing the workshops; Ioanna Baldini and Livio Soares (IBM TJ Watson) for putting together a great set of tutorials; Matt Might (U. Utah) for managing the many complexities of the student travel grant process; John Regehr (U. Utah) for organizing the lightning and poster sessions; Ani Udipi (ARM) for handling registration; Luis Ceze and Karin Strauss for enthusiastically leading the WACI session; Niti Madan (Oracle) for taking care of publicity; Rakesh Komuravelli and Hyojin Sung (UIUC) for managing the submission site; Manju Shevgoor (U. Utah) for developing and maintaining the conference website; and the ASPLOS Steering Committee for their wise input throughout the past year. Sarita's message will acknowledge a number of others who significantly contributed to the technical program.

We are grateful for our many supporters. Their support goes a long way to reducing registration fees, and defrays student costs. This year's supporters are: Microsoft Research, ARM, Google, AMD, HP, VMWARE, Facebook, Intel, Oracle, IBM, and EMC. We also receive generous sponsorship from ACM SIGARCH, SIGOPS, SIGPLAN and NSF for student travel grants.

Finally, thanks to the attendees. You are the lifeblood of the conference. We sincerely hope that you find the program exciting as well as thought provoking. We have a packed program with 49 technical papers, a reception, banquet, business meeting, two keynotes, a debate, and a WACI session. We hope this provides a stimulating platform for several rewarding interactions.

Rajeev Balasubramonian ASPLOS 2014 General co-Chair
Al Davis ASPLOS 2014 General co-Chair 


\section{Program Chair's Message}

It has been a privilege to serve as the program chair for ASPLOS 2014. I am inspired and humbled by the breadth and quality of the work submitted and the commitment of the organizing team to an excellent conference.

The broad goal of a conference is to move the field forward. I was fortunate that recent PC chairs of ASPLOS led very successful conferences, and I mostly followed in their footsteps. Nevertheless I identified two narrower goals for additional efforts: (1) to continue to enhance the image of ASPLOS as a broad, multidisciplinary conference, and (2) to continue to raise the bar for quality and fairness in our review process. This document describes the processes I used, summarizes the program, and acknowledges those who made this program possible.

\section{Scope of ASPLOS}

The call for papers emphasized that ASPLOS is a broad multidisciplinary conference open to new and non-traditional systems related topics. I reached out to "non-traditional" SIGs and other organizations to advertise the call. One concrete outcome is that ASPLOS is "in-cooperation" with SIGBED this year. I hope our ties with SIGBED continue to strengthen in the coming years.

The call also said that papers should emphasize synergy of at least two ASPLOS disciplines (broadly defined) and the submission form explicitly asked authors to identify these areas.

We received 217 submissions, a 12\% increase from last year and a new record. About 147 papers identified architecture as one of their areas, 89 identified PL or compilers, and 94 identified OS. Many papers identified other broad areas, including verification, graphics, big data, networks, cloud computing, mobile computing, embedded systems, software engineering, and more.

For specific research topics, again there was a large diversity. The following were listed by more than 20 submissions: power/energy/thermal management (34 papers), parallel architecture (31), heterogeneous architectures and accelerators (28), caches (27), high-performance computing (26), OS scheduling and resource management (26), compiler optimization (23), software reliability (23), virtualization (22), parallel programming languages (21), and programming models (21). The accepted papers constitute a similarly diverse set of topics.

In summary, ASPLOS continues to live up to its vision of a multidisciplinary conference, attracting a broad range of systems related researchers.

\section{The Review Process: Setting the Stage}

I was fortunate to have an excellent program committee (PC) of 35 members and external review committee (ERC) of 69 members. An additional 43 reviewers contributed to the process.

Review process overview: I used a two phase process similar to recent ASPLOS conferences. Unlike ASPLOS'13 but like other ASPLOS conferences, I obtained phase 2 reviews for only a subset of papers. The two review phases were followed by an author response period, an intense 2week long online discussion period, and a 1-day in-person PC meeting. ERC reviewers were required to participate in all parts of this process except for the $\mathrm{PC}$ meeting.

Aids for reviewer assignments: I assigned all reviews. To help find the best reviewers, I used the following aids new to ASPLOS. (1) The submission instructions allowed unlimited pages for the bibliography and required all citations to include all authors (i.e., no et al.). This borrows from NSF and makes it easier to identify potential reviewers from related work. (2) I asked reviewers to 
suggest other reviewers as part of their review, borrowed from ISCA'13. (3) I allowed authors to (optionally) suggest reviewers.

I required all reviewers to write their assigned reviews themselves. Consulting with others for small aspects was allowed if it brought clear added value (after checking with me for conflicts), but the assigned reviewers needed to write the bulk of the review and give their own scores.

System enhancements for conflicts: I used double-blind reviewing and handled conflicts of interest using community norms. Sandhya Dwarkadas handled the 20 papers with which I had a conflict using the same process described here. I requested Eddie Kohler, the author of the HotCRP reviewing software, to add a new mechanism to handle PC chair conflicts. This mechanism allows the PC chair to yield (most) chair privileges to a different "manager" for a given paper, and allows reviewers to email paper-specific issues to the (anonymous) paper manager. This system (1) significantly streamlined handling the review process for Sandhya, (2) was better at hiding sensitive information from me than alternatives I had previously used, and (3) was better at hiding the conflict from assigned reviewers, preventing inadvertent guessing of author identity. I am grateful to Eddie for implementing such an intrusive change at very short notice.

Tone: I consistently set a tone that encouraged reviewers to not look for perfection but to focus on moving the field forward while being constructive and fair to all authors. I emphasized that there was no target acceptance rate and all worthy papers would be accepted. Accepting many papers implies multitrack sessions and potentially reduced interaction. To mitigate these downsides, the first day of the conference features lightning presentations and the second day features a poster session for all papers, borrowed from MICRO'12. Although I used numeric scores in the review forms, decisions were made based on the review texts.

\section{Phase 1 Reviews}

I assigned 2 PC and 1 ERC reviewers for each paper in phase 1. I received virtually all reviews within two days of the completion deadline (I learned to nag). Unlike ASPLOS'13 (but like other conferences), I did not move all papers to phase 2. An important benefit of two phase reviewing is to relieve reviewers of the burden of reviewing clearly weak papers. On the other hand, a process that rejects papers on the basis of just three reviews risks inadequately informed decisions and inadequate author feedback. I put significant effort to balance these opposing demands.

I classified papers with at least one accept score for overall merit to move to phase 2 by default. Papers with all reject scores with high confidence levels were candidates to not move to phase 2. I highlighted the remaining 38 papers (no accepts, but some undecided and/or low confidence scores) for online discussion among reviewers, using the HotCRP comments system which provides a persistent record. Many reviewers voluntarily discussed many other papers as well.

In parallel, I read most reviews and every review of every paper that was a candidate for terminating at phase 1 or under discussion. For each review that did not provide adequate justification for a low score (e.g., a subjective claim that the paper was incremental without adequate citations of prior work), I used a HotCRP comment to request the reviewer to update their review or risk the paper moving to phase 2. I ensured all score changes were accompanied with justifications in the review text and not just an easy reaction to adjust to the majority. I used the HotCRP color tags feature extensively to help reviewers and myself distinguish different paper categories and track online discussion results.

After this process, all papers with at least one accept or undecided score proceeded to phase 2. A few papers with all reject scores also moved to phase 2 if the review text did not justify the reject 
scores or had low confidence scores (when in doubt, I took the authors' perspective and moved the paper to phase 2). $30 \%$ of the papers did not move to phase 2 .

\section{Phase 2 Reviews}

The phase 1 discussions and reviewer suggestions were very helpful in assigning reviewers for phase 2. All phase 2 papers had at least 5 total reviews, with at least 3 from the PC. The maximum total number of reviews for a PC or ERC member was 19 or 7 respectively.

Mid-phase 2, I used a tool by Andrew Myers ${ }^{1}$ to calibrate excessive negativity or exuberance among reviewers. I sent each reviewer their estimated bias score and an anonymized list of all reviewers' scores. A positive (negative) bias indicated a tendency to give scores that are higher (lower) than others who reviewed the same paper. I believe (hope) this calibration motivated some reflection as reviewers moved to the critical decision-making stages.

The phase 2 reviews were due three days before the author response period. I wanted to ensure high quality reviews to facilitate responses focused on substantive issues. Since there was limited time, I distributed the quality assurance task among all reviewers. The last reviewer that submitted the review on a paper was asked to do a review sufficiency check (RSC) to ensure that (1) the reviews provided sufficient information to make an informed decision for the paper and (2) the reviews provided sufficient feedback to the authors. If the paper passed the RSC, the reviewer colored the paper purple (using HotCRP color tags); otherwise, they noted the problems.

The RSC mechanism significantly enhanced my ability to find the problem papers and focus my attention on them. Many reviews were updated and we identified 18 papers for additional reviews. When the author response started, all reviews from phase 2 had already been received (I got good at nagging). All post-phase2 (post-RSC) reviews had been requested and some were already received! The authors were notified that all pending reviews were late requests and they wouldn't be penalized for not responding to them. At the end, we received a total of 902 reviews.

\section{Online Discussion After Author Response}

This was the most critical phase of the review process. The goal for the online discussion phase was to triage papers into preliminary accept (tagged green, to be presented quickly at the PC meeting), preliminary reject (tagged red, not to be presented at the PC meeting), and discuss (tagged yellow, and the focus of most of the PC meeting).

Each paper was assigned a lead from the PC or ERC to initiate discussion among its reviewers (tagging the paper purple) and lead it towards a consensus (unanimous agreement) for green (accept) or red (reject). If significant discussion did not lead to a unanimous agreement, the paper was marked yellow (discuss). For these, the discussion was expected to reconcile as many differences among the reviewers as possible, leaving only a few substantive differences for a focused PC meeting discussion. That is, tagging yellow was not simply a way to duck work during this phase. These decisions assumed liberal use of shepherding, but not for adding new results.

I monitored discussions for all papers, nagging reviewers, clarifying policies, reminding philosophies, and obtaining yet more reviews as needed, while paying extra attention to papers turning red and those leaning to yellow.

\footnotetext{
${ }^{1}$ http://www.cs.cornell.edu/andru/reviewAdj/
} 
Although this phase required significant effort, it played a critical role in running a smooth PC meeting with well-informed decisions. No system is perfect, but I identify the following advantages of having an online discussion phase with mandatory participation from all reviewers.

(1) It ensured non-PC reviewers' opinions were adequately represented during the $\mathrm{PC}$ meeting. In a conference as broad as ASPLOS, often the non-PC reviewers provide key expertise. We accepted some papers where the only champions were ERC reviewers because they forcefully argued for the paper during the online discussion phase.

(2) It allowed reviewers to reflect on each others' opinions in ways not possible when the first discussion is at the PC meeting; e.g., by reading previous work or confirming an opinion from another expert. These reflections resolved many differences among reviewers that could have become unhelpful sticking points in the limited time of a PC meeting.

(3) It allowed me to provide clarifications on matters of policy and philosophy that sometimes needlessly take up too much PC meeting time (e.g., does a prior workshop submission constitute prior art or does the paper meet the multidisciplinary criteria of ASPLOS).

(4) Mandatory participation (and judicious nagging) reduced (hopefully eliminated) the number of PC members arriving at the meeting without reading author responses and other reviews.

(5) It resulted in a more effective PC meeting with more engagement from all members. I reminded the PC that most of the meeting was focused on papers where $\geq 5$ reviewers had failed to reach consensus after significant deliberation, elevating the importance of their input with clear issues to debate.

This phase identified 23 green and 65 yellow papers for the PC meeting.

\section{PC Meeting}

The PC meeting took place at the O'Hare Hilton on October $30^{\text {th }}$ (a week day) from $8 \mathrm{am}$ to $6: 15 \mathrm{pm}$. One PC member could not attend due to an injury. I made all papers and reviews available to all PC members (sans conflicts), assigned a PC lead to each green and yellow paper, and sent all meeting procedures two days before the meeting. A PC member was allowed to turn any paper to yellow to discuss in the meeting.

The guiding philosophy for the PC discussion was to seek consensus rather than make decisions through narrow majority votes and to give the reviewers (who had read the papers) priority in making the final decision.

For discussion order, within each color, I mostly used the average overall merit score, but I interleaved sessions of green and yellow papers in the morning. We covered about 50 papers before lunch, with the green papers taking little time. For the yellow papers, if about 5 minutes of discussion did not reveal a path to consensus among the reviewers, then we took a full PC (without the reviewers) vote. This vote was advisory input for the reviewers and was recorded in HotCRP. In many cases, the PC vote changed some reviewers' minds and resulted in an immediate consensus decision among the reviewers. In 9 cases, the differences remained and the paper was tabled.

For tabled papers, the reviewers and any interested PC members got together during the ample breaks (with food!) to attempt a consensus. I nudged these small-group discussions along as needed; many were long and intense. A late afternoon session revisited these papers with the full PC. If the reviewers had reached the same agreement as the majority in the previously recorded PC vote, then this result was simply recorded as the final decision. If the new reviewer consensus 
was different from the $\mathrm{PC}$ vote, then the reviewers provided their rationale, entertained questions from the $\mathrm{PC}$, and the new consensus vote was recorded as the final decision. If the reviewers could not reach a consensus even after all the deliberation, then we used the majority vote among them as the final decision. A tie among reviewers used the earlier full PC majority vote for the decision.

During the meeting, a PC member conflicted with a paper left the room for that paper (Sandhya led the discussion for my conflicts). Papers of PC members were handled similar to the others.

We accepted 49 papers, 12 of which were shepherded. The acceptance rate of $23 \%$ was coincidentally the same as last year. 10 accepted papers were co-authored by a PC member out of 24 PC submissions. For most papers and all rejected papers that had an author response, the lead for the paper included a brief summary of the online and PC meeting discussions with the review.

\section{Reflections}

In the last several years, our reviewing processes have come a long way, but there appears to still be scope for improvement. My review checks and the distributed review sufficiency checks (RSCs) resulted in many reviews being updated. The mandatory online discussions, adopted by some but not all recent conferences, clarified many misunderstandings and changed opinions. I believe the high quality of these pre-PC meeting deliberations and the consensus-driven approach led to more informed (and efficient) decisions during the PC meeting. For the papers where consensus was hardest, the face-to-face, small group deliberations during the $\mathrm{PC}$ meeting provided opportunity for more reflection before a final decision.

I would like to credit all past PC chairs from whom I liberally borrowed many ideas. Specifically, Ras Bodik (ASPLOS'13) first exposed me to a review process that employed intensive online discussion and was consensus-driven. The ASPLOS' 14 process was mostly based on his framework. I also benefited from discussions with Vikram Adve (ASPLOS'10), Christos Kozyrakis (MICRO'13), Margaret Martonosi (ISCA'13), and Onur Mutlu (MICRO’12).

\section{The Program}

The 49 accepted technical papers are organized in two tracks. Monday morning will feature two lightning sessions with overviews of all papers to help you choose the sessions to attend. For closer interaction with authors, Tuesday afternoon features a poster session with all papers.

Several plenary sessions complement the technical papers. Monday morning kicks off with a keynote by Brad Calder (Microsoft) describing his experiences with a modern commercial cloud operating system, a topic that directly resonates with many ASPLOS submissions. Tuesday morning starts with a keynote by Jeff Gehlhaar (Qualcomm) describing a brain-inspired processor that overcomes the obstacles of post-Dennard scaling but creates new challenges that could inspire many future ASPLOS submissions. Tuesday ends with the ASPLOS tradition of a "Wild and Crazy Ideas (WACI)" session. The WACI team, ably led by Luis Ceze and Karin Strauss, has done a remarkable job attracting frontier ideas that are sure to inspire, with a cornerstone invited WACInote by Josh Smith (U Washington) on RF-powered computing and communication. Wednesday will start with a debate on whether specialized systems will supplant general-purpose alternatives, moderated by David Wood, again a hot topic touching many ASPLOS submissions.

This year, ASPLOS will have a business meeting and an awards lunch for the first time. Also new is that the technical papers will be openly available in the ACM digital library about a week before the conference. In spite of the packed program, we have included many breaks and a one-of-a-kind excursion - we hope these enable serendipitous interactions leading to technical inspirations and collaborations that make some of the most memorable conference experiences. 


\title{
Acknowledgements
}

The program is the result of the hard work of many people, several of whom went above and beyond the call of duty. The general co-chairs, Rajeev Balasubramonian and Al Davis, were an absolute pleasure to work with. I started to imagine them with a magic wand in their hands - no matter what I asked of them, they said yes, even though it meant more fund raising and attention to more countless details.

I benefitted throughout from the wisdom and quick response times of the steering committee, and earlier mentioned my gratitude to past PC chairs. The submission chairs, my students Rakesh Komuravelli and Hyojin Sung, tirelessly and cheerfully took care of the submission web site. They also attended the PC meeting, recording decisions, moving conflicts in and out, and handling numerous logistics required for a smooth meeting. The review process I described would not be possible without HotCRP and the generosity of its author, Eddie Kohler. Sandhya Dwarkadas handled my conflicts, diligently following all the details of this year's review process. I was originally undecided on whether to have a WACI session, but the infectious enthusiasm of Luis Ceze and Karin Strauss and their volunteering to lead the charge enabled me to make the right decision. David Wood agreed to organize the debate even though I interrupted his vacation. Manju Shevgoor diligently kept the conference web site up to date and Niti Madan contacted many places new to ASPLOS as publicity chair. Michelle Osborne, my assistant, helped with logistics for the PC meeting.

Finally, none of this would be possible without the tireless efforts of the PC, ERC, and other reviewers and the work of the authors. Many of these were truly inspiring.

\author{
Sarita Adve \\ ASPLOS'14 Program Chair \\ University of Illinois at Urbana-Champaign
}




\section{Table of Contents}

Keynote

Session Chair: Al Davis (University of Utah)

- Inside Windows Azure: The Challenges and Opportunities of a Cloud Operating System 1 Brad Calder (Microsoft)

\section{Session 1A: Data Centers}

Session Chair: Anand Sivasubramaniam (Pennsylvania State University)

- Scale-Out NUMA

Stanko Novaković, Alexandros Daglis, Edouard Bugnion, Babak Falsafi

(École Polytechnique Fédérale de Lausanne), Boris Grot (University of Edinburgh)

- Rhythm: Harnessing Data Parallel Hardware for Server Workloads Sandeep R. Agrawal, Valentin Pistol, Jun Pang (Duke University), John Tran, David Tarjan (NVIDIA), Alvin R. Lebeck (Duke University)

\section{Session 1B: Approximate Computing}

Session Chair: Luis Ceze (University of Washington)

- Paraprox: Pattern-Based Approximation for Data Parallel Applications .35 Mehrzad Samadi, Davoud Anoushe Jamshidi, Janghaeng Lee, Scott Mahlke (University of Michigan)

- Uncertain $<$ T $>$ : A First-Order Type for Uncertain Data. James Bornholt (Australian National University), Todd Mytkowicz, Kathryn S. McKinley (Microsoft Research)

\section{Session 2A: Security}

Session Chair: Yuanyuan Zhou (University of California, San Diego)

- Using ARM TrustZone to Build a Trusted Language Runtime for Mobile Applications .

Nuno Santos (INESC-ID \& University of Lisbon),

Himanshu Raj, Stefan Saroiu, Alec Wolman (Microsoft Research)

- Virtual Ghost: Protecting Applications from Hostile Operating Systems. John Criswell, Nathan Dautenhahn, Vikram Adve (University of Illinois at Urbana-Champaign)

- Sapper: A Language for Hardware-Level Security Policy Enforcement 97 Xun Li (Facebook), Vineeth Kashyap (University of California, Santa Barbara), Jason K. Oberg (University of California, San Diego), Mohit Tiwari (University of Texas, Austin), Vasanth Ram Rajarathinam (AMD), Ryan Kastner (University of California, San Diego), Timothy Sherwood, Ben Hardekopf, Frederic T. Chong (University of California, Santa Barbara)

- Finding Trojan Message Vulnerabilities in Distributed Systems 113 Radu Banabic, George Candea, Rachid Guerraoui (École Polytechnique Fédérale de Lausanne)

\section{Session 2B: Resource Management}

Session Chair: Tim Harris (Oracle Labs)

- Quasar: Resource-Efficient and QoS-Aware Cluster Management 127 Christina Delimitrou, Christos Kozyrakis (Stanford University)

- REF: Resource Elasticity Fairness with Sharing Incentives for Multiprocessors 145 Seyed Majid Zahedi, Benjamin C. Lee (Duke University) 
- Price Theory Based Power Management for Heterogeneous Multi-Cores

Thannirmalai Somu Muthukaruppan, Anuj Pathania, Tulika Mitra (National University of Singapore)

- Underprovisioning Backup Power Infrastructure for Datacenters

Di Wang (The Pennsylvania State University), Sriram Govindan (Microsoft Corporation),

Anand Sivasubramaniam (The Pennsylvania State University),

Aman Kansal, Jie Liu, Badriddine Khessib (Microsoft Corporation)

\section{Session 3A: Software Reliability and Testing I}

Session Chair: Santosh Nagarakatte (Rutgers University)

- Comprehending Performance from Real-World Execution Traces: A Device-Driver Case 193

Xiao Yu (North Carolina State University), Shi Han (Microsoft Research), Dongmei Zhang (Microsoft Research), Tao Xie (University of Illinois at Urbana-Champaign)

- Leveraging the Short-Term Memory of Hardware to Diagnose Production-Run Software Failures

Joy Arulraj, Guoliang Jin, Shan Lu (University of Wisconsin-Madison)

- RelaxReplay: Record and Replay for Relaxed-Consistency Multiprocessors

Nima Honarmand, Josep Torrellas (University of Illinois at Urbana-Champaign)

- Prototyping Symbolic Execution Engines for Interpreted Languages

Stefan Bucur (École Polytechnique Fédérale de Lausanne),

Johannes Kinder (École Polytechnique Fédérale de Lausanne \& Royal Holloway, University of London),

George Candea (École Polytechnique Fédérale de Lausanne)

\section{Session 3B: Heterogeneous Computing}

Session Chair: Debbie Marr (Intel Corp.)

- Q100: The Architecture and Design of a Database Processing Unit

Lisa Wu, Andrea Lottarini, Timothy K. Paine, Martha A. Kim, Kenneth A. Ross (Columbia University)

- DianNao: A Small-Footprint High-Throughput Accelerator for Ubiquitous Machine-Learning ...269 Tianshi Chen, Zidong Du, Ninghui Sun, Jia Wang, Chengyong Wu, Yunji Chen (Institute of Computing Technology), Olivier Temam (Inria, France)

- K2: A Mobile Operating System for Heterogeneous Coherence Domains 285 Felix Xiaozhu Lin, Zhen Wang, Lin Zhong (Rice University)

- Disengaged Scheduling for Fair, Protected Access to Fast Computational Accelerators 301 Konstantinos Menychtas, Kai Shen, Michael L. Scott (University of Rochester)

\section{Keynote}

Session Chair: Rajeev Balasubramonian (University of Utah)

- Neuromorphic Processing: A New Frontier in Scaling Computer Architecture Jeff Gehlhaar (Qualcomm Technologies, Inc.)

\section{Session 4A: Virtualization}

Session Chair: Carl A. Waldspurger (independent)

- I/O Paravirtualization at the Device File Boundary Ardalan Amiri Sani, Kevin Boos, Shaopu Qin, Lin Zhong (Rice University)

- KVM/ARM: The Design and Implementation of the Linux ARM Hypervisor 333 Christoffer Dall, Jason Nieh (Columbia University)

- VSWAPPER: A Memory Swapper for Virtualized Environments. 349 Nadav Amit, Dan Tsafrir, Assaf Schuster (Technion - Israel Institute of Technology)

- Cider: Native Execution of iOS Apps on Android. 367 Jeremy Andrus, Alexander Van't Hof, Naser AlDuaij, Christoffer Dall, Nicolas Viennot, Jason Nieh (Columbia University) 


\section{Session 4B: Transactional Memory and Consistency}

Session Chair: Sandhya Dwarkadas (University of Rochester)

- SI-TM: Reducing Transactional Memory Abort Rates through Snapshot Isolation.

Heiner Litz, David Cheriton (Stanford University), Amin Firoozshahian, Omid Azizi (HICAMP Systems), John P. Stevenson (Stanford University)

- Transactionalizing Legacy Code: An Experience Report Using GCC and Memcached

Wenjia Ruan, Trilok Vyas, Yujie Liu, Michael Spear (Lehigh University)

- Fence-Free Work Stealing on Bounded TSO Processors.

Adam Morrison (Technion - Israel Institute of Technology), Yehuda Afek (Tel Aviv University)

- Heterogeneous-race-free Memory Models.

Derek R. Hower (AMD Research), Blake A. Hechtman (AMD Research \& Duke University),

Bradford M. Beckmann, Benedict R. Gaster (AMD Research),

Mark D. Hill (University of Wisconsin-Madison \& AMD Research), Steven K. Reinhardt (AMD Research),

David A. Wood (University of Wisconsin-Madison \& AMD Research)

\section{Session 5A: Storage Systems}

Session Chair: Martha Kim (Columbia University)

- Triple-A: A Non-SSD Based Autonomic All-Flash Array for High Performance Storage Systems 441 Myoungsoo Jung, Wonil Choi (The University of Texas at Dallas),

John Shalf (Lawrence Berkeley National Laboratory), Mahmut Taylan Kandemir (The Pennsylvania State University)

- NVM Duet: Unified Working Memory and Persistent Store Architecture.

Ren-Shuo Liu, De-Yu Shen, Chia-Lin Yang, Shun-Chih Yu (National Taiwan University),

Cheng-Yuan Michael Wang (Macronix International Co., Ltd.)

- SDF: Software-Defined Flash for Web-Scale Internet Storage Systems Jian Ouyang, Shiding Lin (Baidu, Inc.), Song Jiang (Peking University \& Wayne State University), Zhenyu Hou, Yong Wang, Yuanzheng Wang (Baidu, Inc.)

- Integrated 3D-Stacked Server Designs for Increasing Physical Density of Key-Value Stores Anthony Gutierrez, Michael Cieslak, Bharan Giridhar, Ronald G. Dreslinski (University of Michigan), Luis Ceze (University of Washington - Seattle), Trevor Mudge (University of Michigan)

\section{Session 5B: Parallelism I}

Session Chair: Josep Torrellas (University of Illinois at Urbana-Champaign)

- Deterministic Galois: On-Demand, Portable and Parameterless

Donald Nguyen, Andrew Lenharth, Keshav Pingali (The University of Texas at Austin)

- Energy-Efficient Work-Stealing Language Runtimes Haris Ribic, David Yu (SUNY Binghamton)

- Data-Parallel Finite-State Machines Todd Mytkowicz, Madanlal Musuvathi (Microsoft Research), Wolfram Schulte (Microsoft)

- Challenging the "Embarrassingly Sequential": Parallelizing Finite State Machine-Based Computations through Principled Speculation.

Zhijia Zhao, Bo Wu, Xipeng Shen (College of William and Mary)

\section{Session 6A: Parallelism II}

Session Chair: Babak Falsafi (École Polytechnique Fédérale de Lausanne)

- The Sharing Architecture: Sub-Core Configurability for IaaS Clouds

Yanqi Zhou, David Wentzlaff (Princeton University)

- ASC: Automatically Scalable Computation

Amos Waterland, Elaine Angelino, Ryan P. Adams (Harvard University), Jonathan Appavoo (Boston University), Margo Seltzer (Harvard University)

- The Benefit of SMT in the Multi-Core Era: Flexibility towards Degrees of Thread-Level Parallelism 591 Stijn Eyerman, Lieven Eeckhout (Ghent University) 


\section{Session 6B: Compilers, Optimization, and Co-design}

Session Chair: Keshav Pingali (University of Texas at Austin)

- Finding the Limit: Examining the Potential and Complexity of Compilation Scheduling for JIT-Based Runtime Systems

Yufei Ding, Mingzhou Zhou, Zhijia Zhao (The College of William and Mary),

Sarah Eisenstat (Massachusetts Institute of Technology), Xipeng Shen (The College of William and Mary)

- Speculative Hardware/Software Co-Designed Floating-Point Multiply-Add Fusion

Marc Lupon, Enric Gibert, Grigorios Magklis, Sridhar Samudrala, Raúl Martínez, Kyriakos Stavrou,

David R. Ditzel (Intel Barcelona Research Center)

- Post-compiler Software Optimization for Reducing Energy

Eric Schulte (University of New Mexico), Jonathan Dorn (University of Virginia),

Stephen Harding, Stephanie Forrest (University of New Mexico), Westley Weimer (University of Virginia)

\section{Debate}

Session Chair: David A. Wood (University of Wisconsin-Madison)

- Resolved: Specialized Architectures, Languages, and System Software Should Largely Supplant General-purpose Alternatives within the Next Decade

David A. Wood (University of Wisconsin-Madison),

\section{Session 7A: Software Reliability and Testing II}

Session Chair: Shan Lu (University of Wisconsin-Madison)

- Guardrail: A High Fidelity Approach to Protecting Hardware Devices from Buggy Drivers

Olatunji Ruwase (Microsoft Research), Michael A. Kozuch, Phillip B. Gibbons (Intel Labs Pittsburgh),

Todd C. Mowry (Carnegie Mellon University)

- Low-Level Detection of Language-Level Data Races with LARD

Benjamin P. Wood, Luis Ceze, Dan Grossman (University of Washington)

- EnCore: Exploiting System Environment and Correlation Information for Misconfiguration Detection

Jiaqi Zhang (University of California, San Diego),

Lakshminarayanan Renganarayana, Xiaolan Zhang, Niyu Ge, Vasanth Bala (IBM T.J. Watson Research Center),

Tianyin Xu, Yuanyuan Zhou (University of California, San Diego)

\section{Session 7B: Caches and TLBs}

Session Chair: Kathryn S. McKinley (Microsoft Research)

- High-Performance Fractal Coherence

Gwendolyn Voskuilen, T. N. Vijaykumar (Purdue University)

- Locality-Oblivious Cache Organization Leveraging Single-Cycle Multi-Hop NoCs

Woo-Cheol Kwon (Massachusetts Institute of Technology), Tushar Krishna (Intel Corporation),

Li-Shiuan Peh (Massachusetts Institute of Technology)

- Ubik: Efficient Cache Sharing with Strict QoS for Latency-Critical Workloads

Harshad Kasture, Daniel Sanchez (Massachusetts Institute of Technology)

- Architectural Support for Address Translation on GPUs: Designing Memory Management Units for CPU/GPUs with Unified Address Spaces

Bharath Pichai (Rutgers University), Lisa Hsu (Qualcomm Research),

Abhishek Bhattacharjee (Rutgers University) 


\section{ASPLOS 2014 Organization}

General Chairs: Rajeev Balasubramonian, University of Utah Al Davis, University of Utah

Program Chair: Sarita Adve, University of Illinois at Urbana-Champaign

Finance Chair: Gabriel Loh, AMD Research

Workshop Chair: Venkatesh Akella, University of California, Davis

Tutorial Chairs: Ioana Baldini, IBM TJ Watson Research Center

Livio Soares, IBM TJ Watson Research Center

WACI Chairs: Luis Ceze, University of Washington

Karin Strauss, Microsoft Research

Travel Grant Chair: Matthew Might, University of Utah

Poster/Lightning Session Chair: John Regehr, University of Utah

Registration Chair: Aniruddha Udipi, ARM

Publicity Chair: Niti Madan, Oracle

Web Chair: Manjunath Shevgoor, University of Utah

Submissions Chair: Rakesh Komuravelli, University of Illinois at Urbana-Champaign Hyojin Sung, University of Illinois at Urbana-Champaign

Steering Committee: Ras Bodik, UC Berkeley

Doug Burger, Microsoft

George Candea, EPFL

Timothy Harris, Microsoft

Jeremy Gibbons, Oxford University

Vivek Sarkar, Rice University

Michael Scott, University of Rochester

David Wood, University of Wisconsin

Program Committee: Sarita Adve, University of Illinois at Urbana-Champaign

David F. Bacon, IBM Research

Emery Berger, University of Massachusetts, Amherst

Francois Bodin, Irisa

Calin Cascaval, Qualcomm

Luis Ceze, University of Washington

Brad Chen, Google

Trishul Chilimbi, Microsoft Research

Sandhya Dwarkadas, University of Rochester

Krisztian Flautner, ARM 
Program Committee (continued): Tim Harris, Oracle Labs

Mark Hill, University of Wisconsin

Mary Jane Irwin, Pennsylvania State University

Ravi Iyer, Intel Corp.

Martha Kim, Columbia University

Eddie Kohler, Harvard University

John Kubiatowicz, University of California, Berkeley

David Lie, University of Toronto

Shan Lu, University of Wisconsin

Debbie Marr, Intel Corp.

Margaret Martonosi, Princeton University

Kathryn S. McKinley, Microsoft Research

Santosh Nagarakatte, Rutgers University

Satish Narayanasamy, University of Michigan

Vijay Pai, Purdue University

Keshav Pingali, University of Texas at Austin

Parthasarathy Ranganathan, Google

Karin Strauss, Microsoft Research/University of Washington

Martin Vechev, ETH

Carl Waldspurger, independent

Tom Wenisch, University of Michigan

Emmett Witchel, Univerity of Texas at Austin

Lin Zhong, Rice University

Yuanyuan Zhou, University of California, San Diego

Willy Zwaenepoel, EPFL

External Review Committee: Ole Agesen, VMware

Anastassia Ailamaki, EPFL

Krste Asanovic, University of California, Berkeley

Tom Ball, Microsoft Research

Luiz Barroso, Google

Rajeev Barua, University of Maryland

Ricardo Bianchini, Rutgers University

Rob Bocchino, Jet Propulsion Laboratory

Hans Boehm, HP Labs

Greg Bronevetsky, Lawrence Livermore National Laboratory

David Brooks, Harvard University

Angela Demke Brown, University of Toronto

Mihai Budiu, Microsoft Research

Stephen Chong, Harvard University

Albert Cohen, INRIA

Srini Devadas, MIT

Dave Dice, Oracle Labs

Chen Ding, University of Rochester

Mattan Erez, University of Texas at Austin

Babak Falsafi, EPFL

Stephen Freund, Williams College

Xin $\mathrm{Fu}$, University of Kansas 
(continued): Maria J. Garzaran, University of Illinois at Urbana-Champaign

Ada Gavrilovska, Georgia Tech

Robert Geva, Intel Corp.

Ashvin Goel, University of Toronto

Steven Gribble, University of Washington

Dan Grossman, University of Washington

Gernot Heiser, NICTA and University of New South Wales

Tony Hosking, Purdue University

Wilson Hsieh, Google

Chris Hughes, Intel Corp.

Hillery Hunter, IBM T. J. Watson Research Center

Rebecca Isaacs, Microsoft Research

Mahmut Kandemir, Pennsylvania State University

Orran Krieger, Boston University

Rakesh Kumar, University of Illinois at Urbana-Champaign

E. Christopher Lewis, VMware

Kai Li, Princeton University

Geoff Lowney, Intel Corp.

Evangelos Markatos, University of Crete

Milo Martin, University of Pennsylvania

Jonathan McCune, Google

Maged M. Michael, IBM T. J. Watson Research Center

Todd Millstein, University of California, Los Angeles

Tipp Moseley, Google

Onur Mutlu, Carnegie Mellon University

Andrew Myers, Cornell University

Edmund Nightingale, Microsoft Research

Li-Shiuan Peh, MIT

Milos Prvulovic, Georgia Tech

Feng Qin, Ohio State University

Ravi Rajwar, Intel Corp.

Pradeep Ramachandran, Intel Corp., India

Lawrence Rauchwerger, Texas A\&M University

Mendel Rosenblum, Stanford University

Karu Sankaralingam, University of Wisconsin-Madison

Simha Sethumadhavan, Columbia University

Mark Silberstein, Technion

Anand Sivasubramanian, Pennsylvania State University

Edward Suh, Cornell University

Steve Swanson, University of California, San Diego

Josep Torrellas, University of Illinois at Urbana-Champaign

Eric Tune, Google

Amit Vasudevan, Carnegie Mellon University

Kaushik Veeraraghavan, Facebook

TN Vijaykumar, Purdue University

Adam Welc, Oracle

Xiaolan Zhang, IBM T. J. Watson Research Center 
Additional Reviewers: Tor Aamodt, University of British Columbia Parosh Abdulla, Uppsala University

Alaa R. Alameldeen, Intel Corp.

Erik Altman, IBM Research

Christopher Batten, Cornell University

Pradip Bose, IBM Research

Robert Cameron, Simon Fraser University

David Christie, AMD

Chita Das, Pennsylvania State University

Nikil Dutt, University of California, Irvine

Jason Flinn, University of Michigan

Ali Ghodsi, University of California, Berkeley

Tony Givargis, University of California, Irvine

Daniel Gmach, HP Labs

Engin Ipek, University of Rochester

Xiaofan Jiang, Intel Labs

Ian Kash, Microsoft Research

Steve Keckler, University of Texas at Austin/Nvidia

Hyesoon Kim, Georgia Tech

David Levinthal, Google

Steve Lumetta, University of Illinois at Urbana-Champaign

Haohui Mai, Hortonworks

John Mellor-Crummey, Rice University

Klara Nahrstedt, University of Illinois at Urbana-Champaign

Dhabaleswar Panda, Ohio State University

Sungwoo Park, Pohang University of Science and Technology

Avi Pfeffer, Charles River Analytics

Eric Phipps, Sandia National Laboratories

Moinuddin Qureshi, Georgia Tech

Vijay Raghunathan, Purdue University

Christian Renner, Hamburg University of Technology

Chris Rossbach, Microsoft Research

Michael L. Scott, University of Rochester

Martin Schulz, Lawrence Livermore National Laboratory

Dan Sorin, Duke University

Herb Schwetman, Oracle Labs

Kai Shen, University of Rochester

Michael Spear, Lehigh University

Petar Tsankov, ETH

Vincent Vanhoucke, Google

Nalini Venkatasubramanian, University of California, Irvine

Vincent Weaver, University of Maine

Yuan Xie, Pennsylvania State University 


\section{ASPLOS 2014 Sponsors \& Supporters}

- SIGARCH

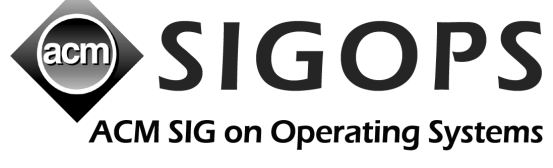

\section{(3im) SIGPLAN}

In co-operation with:

(im SIGBED
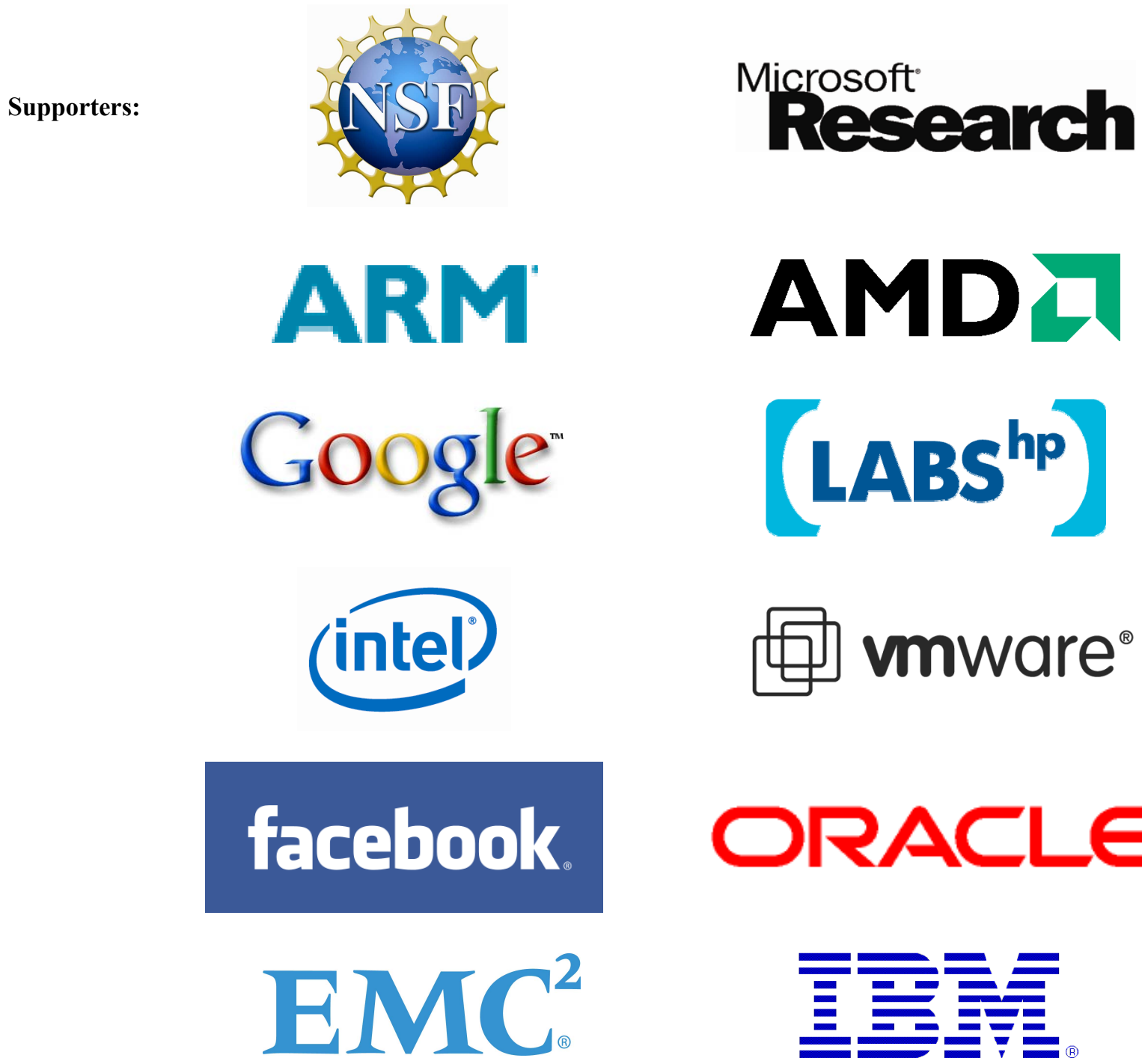

\section{facebook}
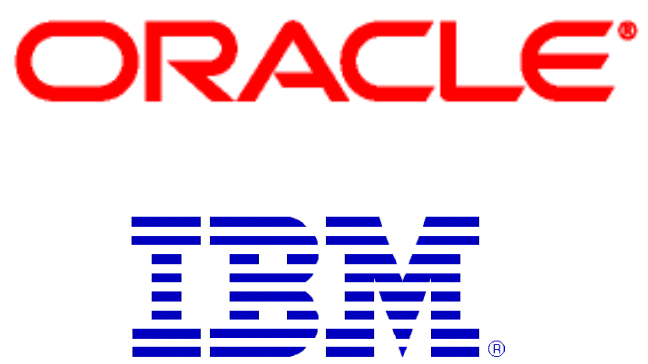